East Eur. J. Phys. 1. 5-12(2021)

East European Journal of Physics

DOI: $10.26565 / 2312-4334-2021-1-01$

PACS: 04.70.Dy, 98.80.-k

\title{
HOW THE LIMIT VALUES WORK
}

\author{
(D) Yuri L. Bolotina, (D) Vladimir V. Yanovsky ${ }^{\mathbf{b}, \mathbf{c}, *}$ \\ ${ }^{a}$ National Science Center "Kharkov Institute of Physics and Technology", 1, Akademicheskaya str., 61108, Kharkiv, Ukraine \\ ${ }^{b}$ Institute for Single Crystals, NAS Ukraine, 60 Nauky Ave., Kharkiv, 61001, Ukraine \\ ${ }^{c}$ V.N. Karazin Kharkiv National University, 4 Svobody Sq., Kharkiv, 61022, Ukraine \\ *Corresponding Author: yanovsky@isc.kharkov.ua, tel.+380-97-614-28-13 \\ Received November 4, 2020; accepted December 15, 2020
}

The efficiency of limiting quantities as a tool for describing physics at various spatio-temporal scales is shown. Due to its universality, limit values allow us to establish relationships between, at first glance, distant from each other's characteristics. The article discusses specific examples of the use of limit values to establish such relationships between quantities at different scales. Based on the principle of reaching the limiting values on the event horizons, a connection was obtained between the Planck values and the values of the Universe. The resulting relation can be attributed to relations of the Dirac type - the coincidence of large numbers that emerged from empirical observations. In the article, the relationships between large numbers of the Dirac type are established proceeding, in a certain sense, from physical principles - the existence of limiting values. It is shown that this ratio is observed throughout the evolution of the Universe. An alternative way of solving the problem of the cosmological constant using limiting values and its relation to the minimum spatial scale is discussed. In addition, a one-parameter family of masses was introduced, including the mass of the Universe, the Planck mass and the mass of the graviton, which also establish relationships between quantities differing by 120 orders of magnitude. It is shown that entropic forces also obey the same universal limiting constraints as ordinary forces. Thus, the existence of limiting values extends to informational limitations in the Universe. It is fundamentally important that on any event horizon, regardless of its scale (i.e., its gravitational radius), the universal value of limit force $c^{4} / 4 G$ is realized. This allows you to relate the characteristics of the Universe related to various stages of its evolution.

KEYWORDS: black hole, event horizon, entropy, limit values

The observed Universe represents a hierarchy of structures with a gigantic spread of characteristic parameters (sizes, energies) from Planck to cosmological. The search for the mysterious connection between large and small scales that describe the world around us is an exciting game of chance. "Random" coincidences caused a storm of enthusiasm and the illusion of unraveling the innermost secrets of nature. The roots of the game go back to antiquity. The first "number theory" - Kabbalah denied the possibility of random coincidences, considering numbers to be a symbol of the spiritual nature of things.

A new era in "physical numerology" began in the first half of the twentieth century, when the characteristic sizes of macro (Universe) and micro (atom, electron) objects became known. The greats entered the game: Weyl [1], Edington [2], Dirac [3].

Already in 1923, in an appendix to his book, "Space, Time, Matter", G. Weyl estimated the ratio of the radius of the Universe to the classical radius of an electron as $\sim 10^{40}$. As he writes, this suggests that the enormous numerical value of the constant is related to the difference in the sizes of the Universe and the electron. P. Dirac belongs to the observation that in the hydrogen atom the ratio of electric to gravitational forces is close to the ratio of the size of the Universe to the classical radius of the electron.

$$
\frac{\text { electrical forces }(H-\text { atom })}{\text { gravitational forces }(H-\text { atom })} \approx \frac{\text { Hubble radius }}{\text { size of electron }}
$$

No physical explanation of this result has yet been found, although it is obvious that any numerological coincidence is of interest only if it can be explained (obtained) in the framework of fundamental physical theory.

The purpose of this article is to introduce a new approach [4] to explain the relationship between different scales. We show that the so-called limit values are an effective tool for achieving this goal. Due to their universality, the limiting values make it possible to establish connections between, at first glance, distant fields of physics.

\section{PHYSICAL MOTIVATIONS}

The traditional formulation of the ultimate goal of physics (in the narrower sense of physical axiomatics) represents the derivation of physical laws from first principles. However, the search for first principles is not inferior in complexity to the original problem. An alternative way to understanding the world around us is to interpret the fundamental limit values as a factor determining the structure of physical laws. Limit values play a central role in both phenomenology and axiomatic theories. It is well known that quantum mechanics can be built on the basis of the existence of a minimum quantum of action $\hbar$, and the special theory of relativity - maximum speed $c$. From relatively recently, it became clear that a similar approach can be implemented in GR, which can be constructed by postulating the existence of a new limit value - the minimum length $[5,6]$ 
Limit values play a key role in the analysis of the measurement process in quantum mechanics. In particular, these quantities are included as parameters in the uncertainty relations, determining the permissible accuracy of measuring physical quantities.

Over the nearly century-long history of its existence, the uncertainty principle (the Heisenberg uncertainty principle (HUP)) has undergone a complex evolution related to the inclusion of gravitational interaction (generalized uncertainty principle (GUP)) and the transition to macroscopic spatial scales (extended uncertainty principle (EUP)) [7-11]

$$
\begin{aligned}
& x \Delta p \geq \hbar \quad(H U P) \\
& \Delta x \Delta p \geq \hbar+l_{P l}^{2} \frac{\Delta p^{2}}{\hbar} \quad(G U P) \\
& \Delta x \Delta p \geq \hbar+l_{P l}^{2} \frac{\Delta p^{2}}{\hbar}+\hbar \frac{\Delta x^{2}}{l_{* l}^{2}} \quad(E U P)
\end{aligned}
$$

Here $l_{*}$ is a new fundamental length scale, the value of which should be determined.

The need to modify the HUP is dictated by the fact that the HUP fails both at short and long distances. The common cause of failure is gravity: a "weak" gravitational interaction can transform into strong either at very small distances (Planck scales), or for very large masses (cosmological scales)

Probably the most important consequence of the transition from the HUP to the GUP is the appearance of a minimum spatial length

and a minimum time scale

$$
\Delta x_{\min }=2 \sqrt{\frac{G \hbar}{c^{3}}}=2 l_{P l}
$$

$$
\Delta t_{\min }=2 \sqrt{\frac{\hbar G}{c^{5}}}=2 t_{P l}
$$

GUP imposes fundamental restrictions on the accuracy $\delta l(\delta t)$ of measuring length (time) $l(t)$ regardless of the measurement method used [12-14]

$$
\delta l \geq\left(l l_{P l}^{2}\right)^{1 / 3}=l_{P l}\left(\frac{l}{l_{P l}}\right)^{1 / 3}, \quad \delta t \geq\left(t t_{P l}^{2}\right)^{1 / 3}=l_{P l}\left(\frac{t}{t_{P l}}\right)^{1 / 3}
$$

The discreteness of space-time generated by the existence of a minimum length and a minimum time is inextricably linked with the concept of an event horizon. The appearance of singularities in the theory is considered to be the first signal that the theory has gone beyond its applicability and needs to be modernized. A natural variant of such modernization is to take into account effects that have fallen from consideration, which allows us to hope (although hopes are not always justified) to make the theory free of divergences. There is also an alternative point of view, formulated by R. Penrose [15] in the form of the principle of cosmic censorship: "Nature has an aversion to naked singularity." According to this principle, the singularities of space-time appear in places that, like the inner regions of black holes, are hidden from the observer. The natural question: is it possible to generalize the principle of cosmic censorship to the level of the "principle of physical censorship", making it a universal physical principle? The existence of a minimum length allows an affirmative answer to this question, and the formation of an event horizon serves as a mechanism for generating a minimum length. In the simplest case, the Schwarzschild solution, the event horizon is a hypothetical sphere around the point of gravitational singularity, dividing the space into two causally unrelated areas. Gravitational radius - the radius of this sphere for a body of mass $m$

$$
r_{g}=\frac{2 m G}{c^{2}}
$$

Now consider the GUP structure

$$
\Delta x \geq \frac{\hbar}{\Delta p}+l_{P l}^{2} \frac{\Delta p}{\hbar}
$$

The right-hand side of this relation represents the sum of two length scales: the wavelength of the observer particle (photon, electron) $\frac{\hbar}{\Delta p}$ and its effective gravitational radius $r_{g}=\frac{m^{*} G}{c^{2}}$, where $m^{*}=E / c^{2}$ ( $E$ is the energy of the observer particle). Such a GUP structure suggests that quantum mechanics and gravity generate two fundamental boundaries for measurement of length: the Compton wavelength $\lambda_{c}=\frac{\hbar}{m c}$ and the gravitational radius corresponding to this mass, and

$$
\frac{r_{g}}{\lambda_{c}}=\left(\frac{m}{m_{P l}}\right)^{2}
$$


In the macrocosm $m \gg m_{P l}$, the Compton wavelength is much smaller than the gravitational radius, which determines the ultimate accuracy in measuring the length. For the Sun $\lambda_{c} \approx 10^{-73} \mathrm{~m}, r_{\mathrm{g}} \approx 3 \mathrm{~km}$. In the microcosm $m \ll m_{P l}$ we are faced with the opposite situation. For the proton $\lambda_{c} \approx 10^{-17} \mathrm{~m}, \quad r_{\mathrm{g}} \approx 10^{-54} \mathrm{~m}$.

An important consequence of GUP is the appearance on the physical scene of a whole set of limit values: minimum length, minimum time, maximum force, maximum power. Consider the nature of the last two quantities. This will help to better understand the role of the event horizon as the boundary of the observed physical reality.

The assertion of the existence of a maximum (limit) force in the framework of GR as a principle was first formulated by G. Gibbons [16]. This force is equal to

$$
F_{\max }=\frac{c^{4}}{4 G} \approx 3.25 \times 10^{43} \mathrm{~N}
$$

The limit does not depend on the nature of the forces and is satisfied for gravitational, electromagnetic, nuclear and any other forces. It is interesting to note that, up to a factor of $2 \pi$, the reciprocal of the ultimate force coincides with the coefficient in front of the pulse energy tensor in the field equations of GR.

The statement about the existence of maximum power is absolutely equivalent to this.

$$
P_{\max }=\frac{c^{5}}{4 G} \approx 9.07 \times 10^{51} \mathrm{~W}
$$

Both of these quantities are components of a 4 -vector $F^{\eta}=\frac{d p^{\eta}}{d t}$. Maximum force and maximum power are invariants: this follows from the invariance of the quantities $\mathrm{c}$ and $\mathrm{G}$. The dependence on time, generally speaking, is not excluded.

Note that the above statements are valid only in space-time dimension $N+1$ for $N=3$ [17]. For $N>3$ maximum force increases with mass as $M^{\frac{N-3}{N-2}}$. As a result, such spatial dimensions allow unlimited gravitational forces. Lack of horizon leads to naked singularities can arise in more than three dimensions

\section{COSMOLOGICAL APPLICATIONS}

The surfaces on which the maximum force (maximum momentum flux) or maximum power (maximum energy flux) is realized are event horizons. Any attempt to exceed the force limit creates a horizon that prevents a further increase in force.

It is fundamentally important that on any event horizon, regardless of its scale (i.e., its gravitational radius), maximum force $c^{4} / G$ is realized. Therefore, for the Planck and current cosmological horizons

$$
G \frac{m_{P l}^{2}}{l_{P l}^{2}} \approx G \frac{M_{U}^{2}}{R_{U}^{2}} \rightarrow \frac{m_{P l}}{l_{P l}} \approx \frac{M_{U}}{R_{U}}
$$

Here $M_{U} \approx 10^{52} \mathrm{~kg}$ is the mass of the observable Universe, and $R_{U}=R_{H}=c H_{0}^{-1} \approx 10^{26} \mathrm{~m}$ is the current Hubble radius. This relation is performed no worse (1).

The accuracy of relation (11) depends on how close the radius of the observable Universe $R_{U}$ is to the radius of the event horizon of mass $M_{U}$. In fact, since $\frac{m_{P l}}{l_{P l}}=\frac{c^{2}}{G}$, it is necessary for (11) to satisfy. $R_{U} \approx \frac{M_{U} G_{U}}{c^{2}}$. To evaluate the fulfillment of this approximate equality, we use the first Friedmann equation

$$
\begin{aligned}
& H^{2}=\frac{8 \pi G}{3} \rho \rightarrow \rho=\frac{3 H^{2}}{8 \pi G} ; \\
& M_{U_{v}}=\frac{4 \pi}{3} R_{H}^{3} \frac{3 H^{2}}{8 \pi G}=\frac{c^{2} R_{H}}{2 G} ; \\
& R_{U}=R_{H}=\frac{2 G M_{U}}{c^{2}}=r_{g}
\end{aligned}
$$

We now consider the asymptotic behavior of the relation $\frac{m_{P l}}{l_{P l}} \approx \frac{M}{R}$ in the framework of SCM for $t \rightarrow \infty$. In this limit

$$
\begin{aligned}
& M_{U}=\frac{4}{3} \pi R_{H}^{3} \rho_{\Lambda}, \quad \rho_{\Lambda}=\frac{\Lambda c^{4}}{8 \pi G}, \\
& R_{H}=\frac{c}{H}=c \sqrt{\frac{3}{\Lambda c^{2}}}=\sqrt{\frac{3}{\Lambda}},
\end{aligned}
$$


Hence

$$
\left.\frac{M_{U}}{R_{H}}\right|_{t \rightarrow \infty} \approx \frac{c^{2}}{G}=\frac{m_{P l}}{l_{P l}}
$$

Those, this ratio is satisfied throughout the evolution of the Universe.

Limit values can be used as an alternative way of finding a solution to the problem of the cosmological constant. For this, the condition for limiting the concentration of energy can be used, in other words, the condition for preventing the formation of an event horizon: the total energy contained in a region with a linear size $L$ should not exceed the mass of a black hole of the same size. If the volume is filled with energy in the form of a cosmological constant, then this condition can be written in the form [18]

$$
L^{3} \rho_{\Lambda} \leq M_{B H} \sim L m_{P l}^{2}
$$

Here $\rho_{\Lambda}$ is the energy density in volume $L^{3}$. If this inequality is violated, a black hole is formed with an event horizon that prevents a further increase in the energy density.

We apply inequality (15) to the Universe as a whole. In this case, it is natural to identify the IR scale with the Hubble radius $H^{-1}$, and $\rho_{\Lambda}$ is understood as density of the dominant component filling the Universe, i.e. dark energy in the form of a cosmological constant. Then for the upper limit of the energy density we find

Given that

$$
\rho_{\Lambda} \sim L^{-2} m_{P l}^{2} \sim H^{2} m_{P l}^{2}
$$

$$
\begin{aligned}
& m_{P l} \simeq 1.2 \times 10^{19} \mathrm{GeV} ; \\
& H_{0} \simeq 1.6 \times 10^{-42} \mathrm{GeV}
\end{aligned}
$$

finally get

$$
\rho_{\Lambda} \sim 10^{-46} \mathrm{GeV}^{4}
$$

This value is close (the difference is only two orders of magnitude and not 120) with the observed density of dark energy. Let us now try at a more fundamental level to understand what our hopes are for solving the problem of the cosmological constant along this path. If we treat the cosmological constant as the energy of zero-point oscillations of a vacuum, then

$$
\begin{aligned}
& \rho_{\text {vac }}=\frac{1}{2} \int_{0}^{\infty} \frac{d \vec{k}}{(2 \pi)^{3}} \sqrt{k^{2}+m^{2}}=\frac{1}{4 \pi^{2}} \int_{0}^{\infty} k^{2} d k \sqrt{k^{2}+m^{2}}, \\
& \rho_{v a c} \sim \frac{k_{\max }^{4}}{16 \pi^{2}} ; k_{\max } \sim M_{P l} \rightarrow \rho_{v a c} \approx 10^{74} \mathrm{GeV}
\end{aligned}
$$

Within the framework of this model, the only way to bring this gigantic value of the density of dark energy closer to the observed one is to reduce the value of ultraviolet cutoff $k_{\max }$ or, what is the same, to degrade the spatial resolution $k_{\max }^{-1}$. Within the framework of the proposed approach, it seems natural to switch from cutoff $k_{\max } \sim m_{P l}$ to cutoff associated with the existence of a minimum length.

In any effective quantum field theory, defined in a spatial region with a characteristic size $l$ and using ultraviolet cutoff $k_{\max }$, entropy is $S \propto l^{3} k_{\max }^{3}$. According to the holographic principle [19], the cutoff value must satisfy the inequality

$$
S \leq S_{B H} \approx\left(\frac{l}{l_{P l}}\right)^{2}
$$

Consider now a size box $l$ (IR-scale) filled with a substance with a UV-cutoff scale $k_{\max }^{-1}$. Then the entropy of such a system $S \propto l^{3} k_{\max }^{3}$ and

$$
l^{3} k_{\max }^{3} \leq\left(\frac{l}{l_{P l}}\right)^{2}
$$

It is natural to identify the UV- scale with a minimum uncertainty of length measurement $\delta l=k_{\max }^{-1}$. In this case, the last ratio is immediately transformed into $\delta l \leq l_{P l}^{2 / 3} l^{1 / 3}$. For $l=R_{u}$ we can determine the scale of UV-cutoff.

According to SCM, quantum mechanics plays a fundamental role in the early Universe, which is described by Planck units built from functional constants $\hbar, c, G$. In the late Universe $(z \rightarrow-1)$, the evolution of the scale factor is determined by the cosmological constant, whose relative density $\Omega_{\Lambda} \rightarrow 1$. A "game" with fundamental constants allows a transition to a new set of Planck variables. Since a cosmological constant plays such a fundamental role in the dynamics of the 
Universe, let us consider the transition from the initial set of Planck units $m_{P l}, l_{P l}, t_{P l}$ built on fundamental constants $\hbar, c, G$ to a new set $m_{\Lambda}, l_{\Lambda}, t_{\Lambda}$ built on constants $\Lambda, c, G[20]$

$$
\begin{aligned}
& m_{\Lambda}=\left(\frac{\hbar_{\Lambda} c}{G}\right)^{1 / 2}=\frac{c^{3}}{G(8 \pi \Lambda)^{1 / 2}}=5.90 \times 10^{56} \mathrm{GeV} ; \\
& l_{\Lambda}=\left(\frac{G \hbar_{\Lambda}}{c^{3}}\right)^{1 / 2}=\left(\frac{8 \pi c^{2}}{\Lambda}\right)^{1 / 2}=4.38 \times 10^{28} \mathrm{~cm} ; \\
& t_{\Lambda}=\left(\frac{G \hbar_{\Lambda}}{c^{5}}\right)^{1 / 2}=\left(\frac{8 \pi}{\Lambda}\right)^{1 / 2}=1.46 \times 10^{18} \mathrm{~S} \\
& \hbar_{\Lambda}=\frac{8 \pi c^{5}}{G \Lambda}
\end{aligned}
$$

The new units perfectly reproduce the mass, size and lifetime of the observed Universe, but solving the problem of the cosmological constant, they give rise to the problem of Planck constant,

$$
\hbar_{\Lambda}=7.35 \times 10^{122} \hbar=7.75 \times 10^{88} \mathrm{~J} \cdot \mathrm{s}
$$

All the same 120 orders! Just the difficulty is "buried" in another place, and, apparently, to solve the problem of the cosmological constant, one should look for fundamentally new approaches.

\section{WEINBERG MASS}

We now turn to the original idea of S. Weinberg [21] to take into account the influence of the dynamics of the Universe at the level of fundamental constants. To do this, he constructed the mass of a hypothetical particle, using, in addition to the fundamental constants $\hbar, c, G$ the Hubble parameter $H$

$$
m_{W}=\left(\frac{\hbar^{2} H}{G c}\right)^{1 / 3}
$$

A set of constants $(\hbar, c, G, H)$, generally speaking, does not provide an unambiguous choice of mass. Therefore, a natural question arises: what physical considerations lead to such a choice of mass?

Surface gravity at the Hubble Horizon

$$
a_{g}=G \frac{M_{U}}{R_{H}^{2}} \approx c H
$$

"Surface" acceleration for a quantum particle of mass $m$ and size $r=\hbar / m c$

$$
a_{g p}=G \frac{m}{r^{2}}==\frac{G m^{3} c^{2}}{\hbar^{2}}
$$

If the Hubble sphere represents the event horizon, and the Weinberg particle is a microscopic black hole, then $a_{g}=a_{g p}$ and

Note that in $\operatorname{SCM} H(t \rightarrow \infty)=\sqrt{\Lambda / 3}$. Hence,

$$
a_{g}=a_{p g} \rightarrow m_{W}=\left(\hbar^{2} H / c G\right)^{1 / 3}
$$

$$
m_{W}(t \rightarrow \infty)=\left(\hbar^{2} \sqrt{\Lambda} / \sqrt{3} c G\right)^{1 / 3}
$$

Weinberg mass admits a curious interpretation [22]. The self-gravitational potential energy $E_{g}$ of this quantum of mass $m$ (and size its Compton wavelength $\hbar / m c$ ) is given by

$$
E_{g}=G m^{2} /(\hbar / m c)=G m^{3} c / \hbar
$$

Substituting (24) into (29) we obtain

$$
E_{g} \approx H \hbar=\hbar c / R_{H}
$$

Since the age of the Universe $1 / H$ which is today the maximum time and, therefore $H$ minimum frequency, the value $E_{g} \approx H \hbar$ can be interpreted as a quantum of the minimum gravitational energy. At a phenomenological level, it is natural to interpret it as the minimum graviton energy.

Let us now consider in more general terms the structure of the set of parameters $(\hbar, c, G, H)$. The most important characteristic of this set is the dimensionless parameter 


$$
I \equiv \frac{c^{5}}{\hbar H^{2} G} \approx 10^{120}
$$

The dimensionless parameter has a simple physical meaning: he represents maximum number of gravitons in the Universe. Indeed,

$$
I=\left(\frac{c^{5}}{G} H^{-1}\right) / \hbar H=\left(P_{\max } H^{-1}\right) / E_{g}
$$

Using a parameter $I$, a one-parameter mass family can be constructed

$$
M_{\gamma}=\frac{\hbar H}{c^{2}}\left(\frac{1}{\hbar H^{2}} \frac{c^{5}}{G}\right)^{\gamma}
$$

From this relation it follows that $m_{W}=M_{1 / 3}$. Note that the Planck mass is also present in this family and corresponds to the choice: $\gamma=1 / 2, M_{1 / 2}=\frac{\hbar H}{c^{2}}\left(\frac{1}{\hbar H^{2}} \frac{c^{5}}{G}\right)^{1 / 2}=\sqrt{\frac{\hbar c}{G}}=m_{P l}$.

The mass spectrum (32) contains four masses that are independent of one of the parameters of the set $(\hbar, c, G, H)$ : the Planck mass $m_{P l}=\frac{\hbar H}{c^{2}} I^{1 / 2}$, the mass of the graviton $\frac{\hbar H}{c^{2}} I^{0}$, the mass of the Universe $M_{\gamma=1}=\frac{c^{3}}{H G}=\left(\frac{c^{5}}{G} H^{-1}\right) / c^{2}$ and $M_{2 / 5}=\left(\frac{\hbar^{3} H}{G^{2}}\right)^{1 / 5}$.

\section{THERMODYNAMIC CONSTRAINTS}

Let us show now that the entropy forces [23] satisfy the same limiting restrictions as ordinary forces. Consider a particle of mass $m$ approaching the event horizon of the black hole of the mass $M(m \ll M)$. On the horizon, this particle will experience acceleration

$$
a=G M m / R_{S h}^{2} m=\frac{c^{4}}{4 G M}
$$

Assume that the force causing the acceleration is of entropic origin. Entropic force is a macroscopic force that arises in systems with many degrees of freedom, as a consequence of the universal tendency to achieve maximum entropy. Entropic force is determined by the condition

$$
F_{\text {ent }}=T \times 2 \pi k_{B} \frac{m c}{\hbar}
$$

Acceleration caused by this force

$$
a_{\text {ent }}=T \times 2 \pi k_{B} \frac{c}{\hbar}
$$

Equating (34) and (36), we reproduce the well-known expression for the temperature of the black hole horizon

$$
T=\frac{\hbar c^{3}}{8 \pi G k_{B} M}
$$

For entropic force, a universal constraint is retained $F \leq \frac{c^{4}}{4 G}$. Really,

$$
F_{\text {ent }}=T \times 2 \pi k_{B} \frac{m c}{\hbar}=\frac{\hbar c^{3}}{8 \pi G k_{B} M} \times 2 \pi k_{B} \frac{m c}{\hbar}=\frac{m}{M} \frac{c^{4}}{4 G}
$$

Since $\frac{m}{M}<1$ then $F_{e n t} \leq \frac{c^{4}}{4 G}$.

Consider a Schwarzschild black hole with parameters $M, R$. Let $R \rightarrow R+l_{\min }=R+2 l_{P l}$. Then $\Delta M=\frac{l_{P l} c^{2}}{G}$. Correspondingly the change in the energy of a black hole

$$
\Delta E=\Delta M c^{2}=\frac{l_{P l} c^{4}}{G}=l_{P l} F_{\max }
$$


The number of bits on the horizon of a black hole of radius $R$

$$
N=\frac{4 \pi R^{2}}{4 l_{P l}^{2}}=\pi \frac{R^{2}}{l_{P l}^{2}}
$$

Increasing the amount of information on the event horizon for $R \rightarrow R+2 l_{P l}$,

$$
\Delta N=4 \pi \frac{R}{l_{P l}}
$$

Let the energy required to create (erase) one bit is $E_{L}$. Then

$$
\Delta E=E_{L} \Delta N
$$

Substituting (39), (41) into (42) we obtain

$$
E_{L}=\frac{l_{P P}^{2} c^{4}}{4 \pi G R}
$$

Given that $R=\frac{2 G M}{c^{2}}$ and $M=\frac{\hbar c^{3}}{8 \pi k_{B} G T}$ finally get

$$
E_{L} \approx k_{B} T
$$

in accordance with the Landauer principle [24].

\section{CONCLUDING REMARKS}

Historical experience teaches that the most interesting discoveries in physics occur during the transition to new characteristic scales of quantities that describe the problem under study. The boundary of the region in which a certain paradigm operates is determined by fundamental constants. For example, the transition from classical mechanics to relativistic is controlled by the speed of light, and the transition from classical mechanics to quantum is controlled by the Planck constant. The transition to the Planck scale is much more complicated, both quantitatively (this area is separated from the parameters currently achieved by tens of orders of magnitude) and qualitatively. (Is the concept of continuous space-time compatible with quantum mechanics). These difficulties make us look for workarounds to solve the problem. One of these areas may be the physics of limit quantities. Some specific examples of the use of limit values were considered in this paper. It is fundamentally important that on any event horizon, regardless of its scale (i.e., its gravitational radius), the universal value of limit force $c^{4} / 4 G$ is realized. This allows you to relate the characteristics of the Universe related to various stages of its evolution.

\section{ORCID IDs}

(D) Yuri L. Bolotin, https://orcid.org/0000-0001-8164-5668; (DVladimir V. Yanovsky, https://orcid.org/0000-0003-0461-749X

\section{REFERENCES}

[1] H. Weyl, Annalen der Physik, 364(10), 101-133 (1919), https://doi.org/10.1002/andp.19193641002.

[2] A. Eddington, Proceedings of the Cambridge Philosophical Society, 27(1), 15-19 (1931), https://doi.org/10.1017/S0305004100009269.

[3] P.A. Dirac, The cosmological constants, Nature, 139, 323 (1937), https://doi.org/10.1038/139323a0.

[4] Yu.L. Bolotin, A.V. Tur, and V.V. Yanovsky, https://arxiv.org/abs/2005.03984v1.

[5] T. Jacobson, Phys. Rev. Lett. 75, 1260 (1995), https://doi.org/10.1103/PhysRevLett.75.1260.

[6] C. Schiller, International Journal of Theoretical Physics, 44, 1629-1647 (2005), https://doi.org/10.1007/s10773-005-4835-2.

[7] G.Veneziano, Europhys. Lett. 2(3), 199 (1986), https://doi.org/10.1209/0295-5075/2/3/006.

[8] E. Witten, Phys. Today, 49, 24 (1996), https://doi.org/10.1063/1.881493.

[9] J. Polchinski, String Theory, (Cambridge University Press, Cambridge, 1998), pp. 402, https://doi.org/10.1017/CBO9780511816079.

[10] R. Adler, P. Chen, and D. Santiago, Gen. Rel. Grav. 33, 2101-2108 (2001), https://doi.org/10.1023/A:1015281430411.

[11] S. Mignemi, Mod. Phys. Lett. A, 25, 1697-1703 (2010), https://doi.org/10.1142/S0217732310033426.

[12] H. Salecker, and E. Wigner, Phys. Rev. 109, 571 (1958), https://doi.org/10.1007/978-3-662-09203-3 15.

[13] F. Karolyhazy, Nuovo Cim. A, 42, 390 (1966), https://doi.org/10.1007/BF02717926.

[14] Y. Ng, Phys. Rev. Lett. 86, 2946 (2002), https://doi.org/10.1103/PhysRevLett.88.139902.

[15] R. Penrose, Ann. N.Y. Acad. Sci. 224, 125-134 (1973), https://doi.org/10.1111/j.1749-6632.1973.tb41447.x.

[16] G. Gibbons, Found. Phys. 32, 1891 (2002), https://doi.org/10.1023/A:1022370717626.

[17] J.D. Barrow, https://arxiv.org/abs/2005.06809v1.

[18] A. Cohen, D. Kaplan, and A. Nelson, Phys. Rev. Lett. 82, 4971 (1999), https://doi.org/10.1103/PhysRevLett.82.4971.

[19] 19.G.'t Hooft, https://arxiv.org/abs/gr-qc/9310026v2.

[20] P-H. Chavanis, Eur. Phys. J. Plus, 129, 222 (2014), https://doi.org/10.1140/epjp/i2014-14222-0.

[21] S. Weinberg, Gravitation and Cosmology, (JohnWiley \& Sons, New York, NY, 1972).

[22] I. Gkigkitzis I. Haranas, and S. Kirk, Astrophys. Space Sci. 348, 553-557 (2013), https://doi.org/10.1007/s10509-013-1581-4.

[23] E.P. Verlinde, On the origin of gravity and the laws of Newton, JHEP 1104:029, 2011, https://doi.org/10.1007/JHEP04(2011)029.

[24] R. Landauer, IBM Journal of Research and Development, 5(3), 183-191 (1961), https://doi.org/10.1147/rd.53.0183. 


\section{ЯК ПРАЦЮЮТЬ ГРАНИЧНІ ЗНАЧЕННЯ}

Ю.Л. Болотін ${ }^{\mathrm{a}}$, В.В. Яновський ${ }^{\mathrm{b}, \mathrm{c}}$

${ }^{a}$ ННЦ "Харківський фізико-технічний інститут" НАН Украӥни, Харків, Украӥна

${ }^{b}$ Інститут монокристалів НАН України, Харків, Україна

' Харківський національний університет імені В.Н. Каразіна, Харків, Украӥна

Показана ефективність граничних величин як інструменту для опису фізики на різних просторово-часових масштабах. Завдяки своїй універсальності, граничні значення дозволяють встановлювати взаємозв'язки між, на перший погляд, далекими один від одного характеристиками. У статті розглянуті конкретні приклади використання граничних значень для встановлення таких зв'язків між величинами на різних масштабах. Виходячи 3 принципу досягнення граничних значень на горизонтах подій отримано зв'язок між планківськими величинами та величинами всього Всесвіту. Отримане співвідношення можна віднести до співвідношень діраківського типу - збігу великих чисел, які з'являлися з емпіричних спостережень. У статті співвідношення між великими числами типу діраківських, встановлюються виходячи, в певному сенсі, 3 фізичних принципів - існування граничних величин. Показано, що це співвідношення дотримується на всьому протязі еволюції Всесвіту. Обговорюється альтернативний спосіб вирішення проблеми космологічної сталої з використанням граничних величин і зв'язок ії̈ з мінімальним просторовим масштабом. Крім цього введено одно параметричне сімейство мас, яке включає в себе масу Всесвіту, масу Планка і масу гравітону, яке також встановлює зв'язок між величинами які відрізняються на 120 порядків. Показано, що ентропійні сили також підкоряються тим же універсальним обмеженням на граничні значення, як $\mathrm{i}$ звичайні сили. Тим самим існування граничних величин поширюється на інформаційні обмеження у Всесвіті. Принципово важливо, що на будь-якому горизонті подій, незалежно від його масштабу (тобто його гравітаційного радіуса), реалізується універсальне значення граничної сили $c^{4} / 4 G$. Це дозволяє зв'язати характеристики Всесвіту, що відносяться до різних етапах іiї еволюції.

КЛЮЧОВІ СЛОВА: чорна діра, горизонт подій, ентропія, граничні значення 